\title{
Multilayer Filters for Extending the Duty Cycle of Optical Telescopes in the Highest Energy Cosmic Ray Experiments
}

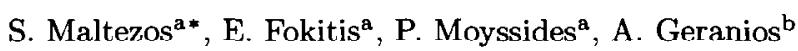 \\ ${ }^{a}$ Physics Department, National Technical University of Athens, \\ 9 IIeroon Polytechniou, GR 15780, Athens, Greece \\ bPhysics Department, University of Athens, \\ Panepistimioupoli, GR 15771, Athens, Greece
}

Multilayer optical filters, appropriately designed to reject the optical noise emitted in the range between the main UV spectral lines of the atmospheric $N_{2}^{+}$fluorescence, induced by the Ultra High Energy Cosmic Rays (UHECR) via the effect of Extensive Air Showers (EAS), are presented in this work. For the design we used the method of simulated annealing, assuming 40 dielectric layers. A simplified model of the main optical noise components has been used for calculating the evolution of the trigger probability and the resulting duty cycle of a pixel detertor. The method has been applied for the fuorescence telessope of the AUGFR Observatory, assuming the use of various optical filters.

\section{INTRODUCTION}

A UHECR particle, typically of energy larger than $10^{19} \mathrm{eV}$, produces an EAS in the atmosphere. Such extremely rare cosmic rays have been observed, but the statistics is still too low to explain a number of cosmological and particle physics questions. The secondaries and their products result in an extremely large number of positrons and electrons, among other particles, participating in the EAS. The electrons and positrons share almost $90 \%$ of the total energy of the shower and induce excited ionized molecular nitrogen states. Their de-excitation leads to the emission of atmospheric fluorescence in the characteristic spectral lines of nitrogen. In the experiments that are based on the above phenomenon, like AUGER [1], HiRes [2] and T $\Lambda[3]$, the nitrogen fluorescence UV radiation is detected by telescopes, typically based on a photomultiplier (PMT) pixel array camera; this is done in order to obtain a longitudinal image of the shower and its time evolution.

In section 2 the principle of operation of the fluorescence telescopes and the filters used are pre-

$\overline{\text { * Corresponding author }}$ sented. A model for the optical noise is described briefly in section 3 . In section 4 the duty cycle of the detector operation is determined while in section 5 conclusions and prospects are given.

\section{FLUORESCENCE TELESCOPES AND OPTICAL FILTERS}

\subsection{Considerations on the telescope per- formance}

The fluorescence telescopes focus the atmospheric fluorescence radiation $\left(N_{2}^{+}\right)$, usually via spherical mirrors, on a 2-dimensional pixel array (each pixel being a PMT) with angular resolution of the order of $1.5 \times 1.5 \mathrm{deg}^{2}$ (Pierre AUGER experiment) or $1 \times 1 \operatorname{deg}^{2}$ (HiRes experiment). Optical UV filters, transparent in the range 300-410 $\mathrm{nm}$, are typically used to efficiently reject the optical noise in the visible spectral range $(\geq 400$ $\mathrm{nm}$ ). The UV component of the noise, which normally remains when using single pass-band filters, could be reduced using notch type optical filters studied in this work.

The filters can be located either in the aperture of the telescope or in front of the pixel array camera. A useful way to describe the performance of 
the pixel detector (consisting mainly of PMT, filter, mirror and other optical components), is to use the following two parameters for the efficiency in detecting the fluorescence signal and the optical noise (background). These are the detection efficiencies $E_{s}$ and $E_{b}$, respectively [4].

\subsection{Multilayer filters and their design}

Multilayer interference filters could provide some significant advantages in comparison with commercial absorption type filters, such as:

I. Higher spectral transmittance on average.

II. Steeper wavelength cut-off.

III. Flexibility to design them according to the desired spectral transmittance.

However, the cost per unit area of these filter types is higher and depends on the total number of layers used. We have being designing such filters using the method of "Simulated Annealing" $[5,6]$ to obtain the optimal layer configuration. Twenty layer pairs of materials of high $\left(\mathrm{Ta}_{2} \mathrm{O}_{5}\right)$ and low $\left(\mathrm{SiO}_{2}\right)$ refractive index were considered to realize two filter types: a) A recently designed multi-band type, which has multiple transmission bands (notch type filter) matching with the fluorescence spectral lines (see Fig. 1). Feasibility study by companies producing such industrial filters is under way. b) A typical single-band design (presented in Ref. [7]). Such single-band multilayer filters, based on a totality of around 56 layers, have been successfully produced commercially and tested [8]. In addition, we compare the above filters with the absorption filter M-UG6 [9], used in the Engineering Array of AUGER fluorescence detector (FD), the one used in the HiRes, and the one proposed for TA (BG3 $6 \mathrm{~mm}$ thick) experiments.

\subsection{Pixel detector sensitivity}

The minimum detectable signal by the pixel detector, $I_{s, \min }$, expresses its sensitivity. This signal is detected with a certain signal-to-noise ratio $n$ and it is assumed being fixed (in the following is taken equal to 5). The calculation of the $I_{s, \min }$ is based on an analysis of the noise fluctuations

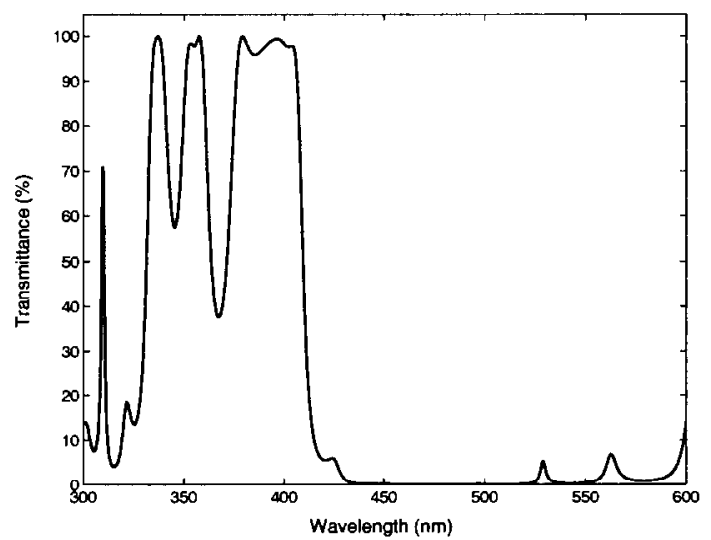

Figure 1. The simulated multi-band (notch) multilayer filter consisting of 40 dielectric layers.

given in Ref. [4] and is given by

$I_{s, \min }=\frac{n^{2}}{2 S_{n} E_{s}}\left(1+\sqrt{1+\frac{8 I_{b} B_{n} E_{b}}{n^{2}}}\right)$

where, $I_{b}$ is the integrated (total) photon flux of the optical noise, and $S_{n}, B_{n}$ are the integral acceptances of the PMT itself in detecting the signal and optical noise, respectively. As seen below the knowledge of $I_{s, \min }$ allows us to calculate the trigger probability of the detector.

\section{MODELLING THE OPTICAL NOISE}

The optical noise is defined as the radiation, within the spectral range of the detector, from UV to visible, which is unrelated to the EAS event. A simplified model for the optical noise has been used including the following main sources:

Nightsky radiation The night sky background radiation is mainly due to stars and interstellar media, as well as to the radiation from the atmosphere itself.

Moonlight The diffused light of the moon. 


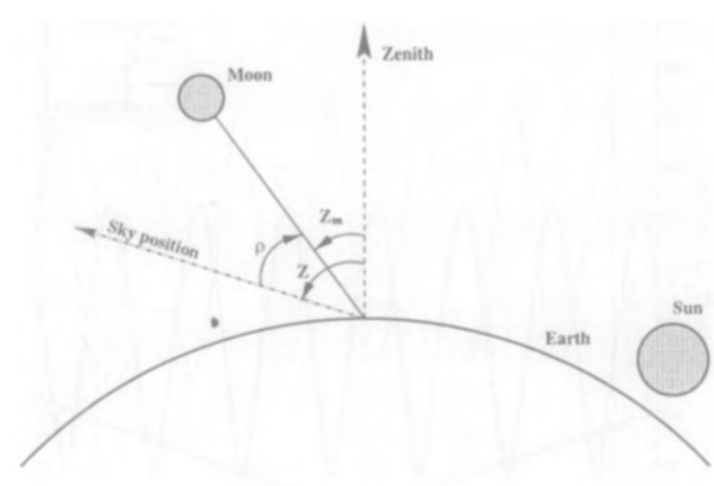

Figure 2. A representative diagram showing the zenith distances of the moon and detector, assuming the phase angle of the moon to be about $40^{\circ}$ ( 3 days after full moon). The relative position of the sun (after its set) is presented as well.

Zodiacal light The zodiacal light is produced by the sun light scattered from the clouds of dust in the space around the earth [10].

For the night sky background radiation we used measurements from "Mauna Kea" [11] converted to units of photon flux. The overall illuminance of the scattered moonlight is given by the expression $[12,7]$ :

$I_{b}(\lambda)=f(\rho) I_{m}(\lambda, \alpha) e^{-\frac{X\left(Z_{m}\right)}{X_{o}(\lambda)}}\left(1-e^{-\frac{X(Z)}{X_{o}(\lambda)}}\right)$

where $Z_{m}$ is the zenith distance of the moon, $Z$ is the zenith distance of the detector (sky position: equal to $\left.74^{\circ}\right), p=\left|Z_{m}-Z\right|$ is the scattering angle in degrees, $f(\rho)$ is the "scattering function" which consists from two terms expressing the Rayleigh and Mie scattering. Also, $X(Z)$ is the optical path length, $X_{o}(\lambda)$ is the absorption path and $\alpha$ is the phase angle of the moon varying from 0 to $180^{\circ}$. The $I_{m}(\lambda)$ expresses the spectral dependence of the illuminance. The spectral distribution used in the above formula is based on experimental data described in Ref. [7].

Astronomical data, concerning the zenith distance of the sun $\left(Z_{s}\right)$ and moon $\left(Z_{m}\right)$, in relation with the phase angle of the moon $(\alpha)$, see Fig.2, have been used for the calculations [13].

The zodiacal light (ZL) illuminance is calculated by assuning for simplicity the same scattering function, considering $\alpha=0$, and $Z_{s}$ instead of $Z_{m}$. For scattering angles of our interest (during the night), where $\rho \geq 34^{\circ}$, and when the line of sight of the telescope is on the helioecliptic plane, the scattering function of this model is consistent with ZL data given in Ref. [14]. We used a normalization factor based on experimental data that the average value of $\mathrm{ZL}$ illuminance is about 4 times than that of nightsky radiation [15]. This means that we follow the general empirical knowledge that the zodiacal light has a spectral distribution quite similar to the one of the sun and the moon. The total illuminance is the sum of the three components described above.

The overall optical noise is shown in Fig.3. This case concerns an angular position of the moon, and such a moon phase angle, resulting in a significant noise level, but reasonably low, to allow the operation of the FD detector. The multiple small peaks in the region about $300-380 \mathrm{~nm}$ and the larger one at $557.7 \mathrm{~nm}$ are due to the emission of the atmospheric oxygen.

\section{DETERMINATION OF THE DUTY CYCLE}

\subsection{Trigger probability and its variation}

According to the energy of the cosmic rays, a power-law spectrum of particle energies is produced [16]. The photon flux is considered to be proportional to the primary energy and, thus, the number of particles which can produce a signal $I_{s}$ (in $\mathrm{ph} / \mu \mathrm{s}$ ) is given by $[7]$ :

$\frac{d N}{d I_{s}}=(\gamma-1) \frac{I_{s}^{-\gamma}}{I_{s, l o w}^{1-\gamma}}$

where $\gamma$ is the "differential spectral index", taken equal to 2.7 [17] for the energy range around $10^{19} \mathrm{eV}$, while the normalization factor is calculated for total probability equal to 1 . The quantity $I_{s, \text { low }}$ represents the lowest value of $I_{s, \min }$ in the case of moonless night sky conditions and assuming the use of a hypothetical ideal notch filter (having bands with transmittance $100 \%$ and dips 


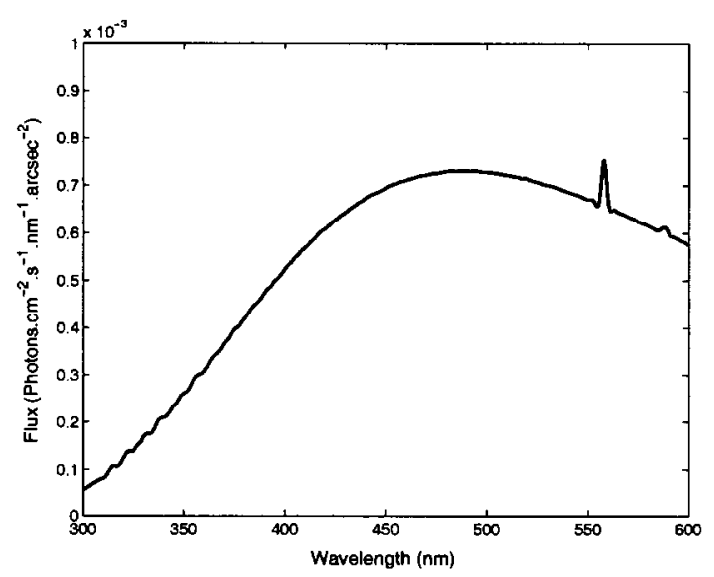

Figure 3. The optical noise spectrum expected for the $22^{\text {th }}$ of .January 2003 a.t 6:40 a.m under crescent moon $\left(Z_{m}=50^{\circ}, \alpha=50^{\circ}\right)$. This spectrum includes the nightsky background radiation taken from ref.[11].

approaching to zero). The value of $I_{s, \text { low }}$, used for the calculations, is $660.6 \mathrm{ph} / \mu \mathrm{s}$. This amount of photon flux, collected by the first pixel of the FD camera, comes from the first segment of a simulated EAS event, produced by a cosmic ray near the energy threshold of the detector, as given by Ref. [18]. The formula used in this reference has been modified in order to use for the fluorescence yield a number of 4.8 photons per $\mathrm{m}$ [19]. For a given detection limit, $I_{s, \min }$, the probability to achieve a trigger to the PMT is calculated by integration of Eq. 3, that is

$P_{t r}=P\left(I_{s} \geq I_{s, \min }\right)=\left(\frac{I_{s, \text { min }}}{I_{s, l o w}}\right)^{1-\gamma}$

The above trigger probability, $P_{t r}$, is a function of time due to the variation with time of the detection limit, $I_{s, m i n}$, according to Eq.1, including the quantities $I_{b}, E_{b}$ and $B_{n}$.

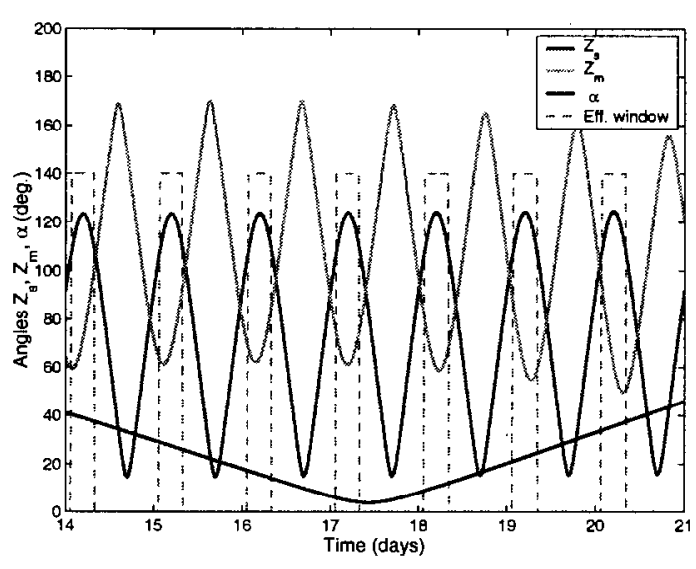

Figure 4. The evolution of the zenith distances of sun and moon and the phase angle of the moon during the week Jan. 15-21/2003. The effective window of operation is shown with the dashed line

\subsection{Expected filter performance for monthly operation}

Below, the method has been applied for the determination of the duty cycle of the FD of the AUGER observatory. The January of 2003, in Malargue of Argentina (Latitude $=28^{\circ} \mathrm{S}$ and Longitude $=34^{\circ} \mathrm{W}$ ), has been selected as a typical example. A software code has been developed to simulate the trigger probability in intervals of $20 \mathrm{~min}$. First, the related zenith distances of the sun and moon, along with the moon phase angle, have been used (see Fig.4) to calculate the optical noise photon flux. The week Jan. 1-7, including new moon and the week Jan. 15-21, including full moon, have been selected for the calculations for comparison reasons.

The time intervals of a 24-hours day for which the zenith distance of the sun is smaller than $108^{\circ}$ (daylight present) have been excluded in the calculations. The obtained $P_{t r}$ has a certain variation during the above weeks, as shown in Figs 5 and 6 . Its average, $P_{t r, a v}$, expressing the trigger 


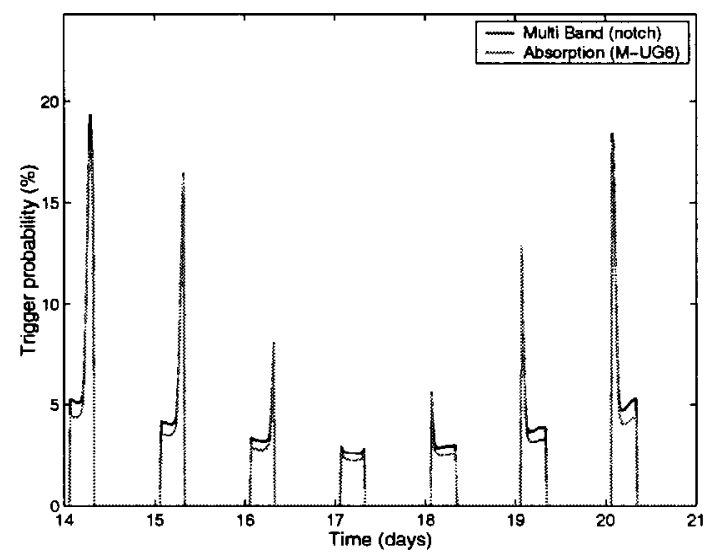

Figure 5. The trigger probability calculated during the week Jan. 15-21/2003, as it is obtained for the notch, and absorption (M-UG6), filter types. Only the effective time window is shown (without sunlight).

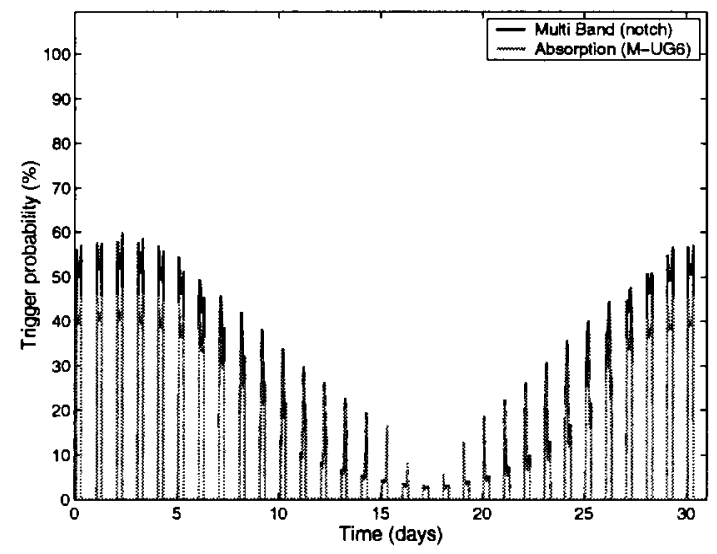

Figure 6. The trigger probability during the whole month, January 2003, as it is obtained for the notch, and absorption (M-UG6), filter types. efficiency, is calculated during the selected time intervals. The duty cycle, $r_{d c}$, is defined as: The fraction of the time intervals of operation, with $P_{t r}$ greater than a certain threshold $p_{t h}$, with respect to the total time interval. This threshold $\left(p_{t h}\right)$ expresses the the minimum trigger probability, to keep the shutter of the detector open. Setting the indicative value of $5 \%$, we find the results given in the table 1 . This value has been selected because it corresponds almost to the mean value of the optimum values for the two compared filters (see section 4.3). However, the comparison of the various filters should be made using a uniform independent probability threshold, which could be set based on economical or practical reasons and not on the filter itself that has been used.

The $r_{d c}$ cannot be greater than a maximum value specified by the presence of the sun during a day. In weekly operation the improvement, using the notch filter with respect to the absorption one M-UG6, is $27.3 \%$ for the week Jan. 1-7, and much higher, $37 \%$, for the week Jan. 15-21. The latter impressive difference, in the $P_{t r, a v}$, is explained by the shape (height and width) of the peaks during the considered week (see Fig. 5).

The uncertainties in these results come mainly: from the uncertainties in the filter and PMT parameters (leading to a relative error of $I_{s, s m i n}$ $4 \%$ ), and also from those of the differential spectral index $\gamma$ (assumed to be 5\%). The resulting error has been estimated to vary from around $7 \%$, for new moon, to around $32 \%$ for full moon.

\subsection{Optimizing the duty cycle}

The FD detector can operate in time intervals where the trigger probability is greater than a selected $p_{t h}$. The value of $p_{t h}$ affects the cost of operation and the life time of the detector. An appropriate performance indicator, $J\left(p_{t h}\right)$, can be used to find the optimal $p_{t h}$. The $P_{t r, a v}$ has to be as high as possible during the operation. On the other hand, it is desirable to minimize the cost of operation per unit trigger probability, which is inversely proportional to the average trigger probability within the particular effective time windows of the selected duty cycle, $P_{t r, a v, p}$. Consequently, a reasonable performance indicator can be defined 
Table 1

Calculated results.

\begin{tabular}{lcccc}
\hline Filter Type & week: Jan. 1-7 & week: Jan. 1-7 & week: Jan. 15-21 & week: Jan. 15-21 \\
& $P_{t r, a v}(\%)$ & $r_{d c}(\%)$ & $P_{t r, a v}(\%)$ & $r_{d c}(\%)$ \\
\hline Ideal (notch) & 14.3 & 24.6 & 1.00 & 10.5 \\
Multi-Band (notch) & 12.6 & 24.6 & 0.78 & 8.7 \\
Single-Band & 11.7 & 24.6 & 0.59 & 5.3 \\
Absorption (M-UG6) & 9.9 & 24.6 & 0.57 & 5.0 \\
Absorption (in HiRes) & 9.0 & 24.6 & 0.53 & 4.8 \\
Absorption (BG3) & 10.0 & 24.6 & 0.43 & 4.4 \\
\hline
\end{tabular}

The classification of various optical filters based on the obtained trigger performance. The weeks Jan. 1-7 and Jan. 15-21, have been used for the calculations, considering an indicative uniform trigger probability threshold $5 \%$.

as:

$J\left(p_{t h}\right) \equiv P_{t r, a v} \cdot P_{t r, a v, p}=P_{t r, a v}^{2} \cdot r_{d c}$

Varying $p_{t h}$ from 0 to $100 \%$ we calculated the function $J$ for each filter type. Below, we compare the notch filter with the M-UG6 one because the latter appear to have best performance among the absorption filters according to the table 1 . Thus, for the notch filter we found an optimal $p_{t h}=3 \%$ with $J_{\max }=7.5 \%$, while the corresponding values for the absorption filter M-UG6 are $6.0 \%$ and $6.7 \%$, respectively. Thus, considering the optimal threshold, an improvement of $12 \%$ in the performance indicator could be accomplished using the notch type filters.

\section{CONCLUSIONS AND PROSPECTS}

Using a simplified model of the optical noise, the trigger probability of the FD detector of AUGER, during the January of 2003 in Malargue of $\Lambda$ rgentina, has bcen studied. Using a recently designed multilayer notch type filter we find that the time depended trigger probability can be improved with this filter in comparison with absorption filters used or proposed for future FD experiments. In addition, during operation under crescent moon, a wider duty cycle of the detector operation can be achieved. For a highly inclined EAS, for which the obtained signal could be low, the trigger probability is also expected to be low, and thus, the improvement may be very significant for the statistics of such cosmic ray events. The presented results have neglected variations on the optical noise due to aerosol presence, aurorae, and variations in the earth's atmosphere radiation (11-year cycle of solar activity).

\section{ACKNOWLEDGMENTS}

We thank Drs R. Korakitis and J. Seiradakis for the appropriate modification of the Sky Map Pro, version 8, and providing us with the astronomical data of angular positions of the sun and moon. We also gratefully acknowledge the stimulating discussions with members of the AUGER collaboration.

\section{REFERENCES}

1. Pierre Auger Project: http://www.auger.org (and Technical info arranged as GAP Notes, therein).

2. T. Abu-Zayyad et al., NIM A 450, pp. 253268 (2000).

3. TA collaboration, "Design report", pp. 27-44 (2000)

4. S. Maltezos, E. Fokitis, GAP note, 2000-012 (2000).

5. P. J. M. van Laarhoven and E. H. I. Aarts, Simulated Annealing: Theory and Applications, Kluwer Academic Publishers (1992). 
6. E. Fokitis, S. Maltezos, E. Papantonopoulos, JHEP, 045 (1999).

7. E. Fokitis, S. Maltezos, P. Moyssides, A. Geranios, presented at the "Natural Radiation Environment (NRE-VII)", in Rhodes, Greece (2002).

8. E. Fokitis et al., Proceedings of $27^{\text {th }}$ ICRC 2001, Hamburg, Germany, pp. 667-670 (2001).

9. C. Boreani et al., IEEE Trans. Nucl.Science, 483, p406 (2001).

10. W. Edward, The Astronomical Journal, 496:1-8 (1998).

11. Typical spectrum of visible night sky emission at Mauna Kea: CFHT Observers' Manual, Sect. 5, http://www.cfht.hawaii.edu/ Instruments/ObserverManual/chapter5.html.

12. K. Krisciunas, B.E. Schaffer, PASP, Vol. 103, pp. 1033-1039 (1991).

13. Chris Marriott, England, software program: Sky Map Pro, version 8.

14. Ch. Leinert et al., Astr. Astroph. Suppl. Ser., 127, pp.1-99 (1998).

15. H. W. Jensen et al, to appear in the SIGGRAPH conference proceedings.

16. A. M. Hillas, Ann. Rev. Astr. Astrophysics 22, 425 (1984).

17. J. W. Elbert, Proceedings of Tokyo Conference on Techniques for the Study Extremely IIigh Energy Cosmic Rays (of M. Nagano), p232 (1993).

18. A. Geranios et al., Proceedings of $27^{\text {th }}$ ICRC 2001, Hamburg, Germany, pp. 671-674 (2001).

19. F. Kakimoto et al., Nucl. Instr. and Meth. A, 372 , pp. 527-533 (1996). 